\title{
A NEW LOOK AT URBAN WATER STORAGE IN A SERIES OF CONNECTED DAMS
}

\author{
PHIL HOWLETT, ${ }^{*}$ University of South Australia \\ CHARLES PEARCE, ${ }^{* *}$ University of Adelaide \\ JULIA PIANTADOSI, ${ }^{* * *}$ University of South Australia
}

\begin{abstract}
We propose a discrete state-space model for storage of urban stormwater in two connected dams using an optimal pump-to-fill policy to transfer water from the capture dam to the holding dam. We assume stochastic supply to the capture dam and independent stochastic demand from the holding dam. We find new analytic formulae to calculate steady-state probabilities for the contents of each dam and thereby enable operators to better understand system behaviour. We illustrate our methods by considering some particular examples and discuss extension of our analysis to a series of three connected dams.
\end{abstract}

Keywords: Urban stormwater; water storage; water distribution; systems of connected dams; Markov decision processes

2010 Mathematics Subject Classification: Primary 60J20

Secondary 15A18; 15B51; 60J22

\section{Introduction}

For much of the 20th century urban drainage systems were designed to remove stormwater from cities and suburbs as quickly as possible. Water was captured from houses and roadsides in underground pipes and large concrete-lined drains which emptied directly into local rivers and streams. Smaller urban streams were often modified to substantially increase flow rates. The enhanced run-off resulted in widespread pollution of urban waterways with waste materials, flooding of low-lying areas when drains became blocked with debris, and damage to adjoining marine environments by repeated incursion of large volumes of freshwater. While society generally has been content to tolerate massive wastage of urban stormwater it has become increasingly obvious in recent times that traditional and existing urban water supply systems are often grossly inadequate. This is particularly true in semi-arid mediterranean climate regions where water-usage restrictions in large cities have now become routine during the hot, dry summer season. See https://www.ladwp.com/ladwp/faces/ladwp/aboutus/a-water/a-w-conservation/aw-c-ordinanceandcodes and www.sawater.com.au/SAWater/Environment/WWM/ for details of typical restrictions. Consequently, community attitudes are changing and progressive local government organisations have established a multitude of small-scale stormwater capture and retention schemes. Underground pipes and concrete drains have been replaced by lakes and reedbeds fed by open watercourses lined with re-introduced endemic vegetation. By incorporating

Received 16 January 2013; revision received 10 May 2013.

* Postal address: Scheduling and Control Group, Centre for Industrial and Applied Mathematics, University of South Australia, Mawson Lakes, 5095, Australia.

** Postal address: School of Mathematical Sciences, University of Adelaide, Adelaide, 5000, Australia.

*** Email address: julia.piantadosi@unisa.edu.au 
natural cleaning mechanisms and slowing down run-off both pollution and flooding have decreased. Some specific results are reported in [4]. Reclaimed stormwater is used by local communities for environmental purposes and by local industry.

\subsection{The Parafield stormwater storage and supply system}

The City of Salisbury in South Australia is a world-leader in the development of urban water resources. The Parafield stormwater management system is one such scheme. Stormwater from an urban waterway is diverted to a $50 \mathrm{ML}$ capture dam and then pumped to a $50 \mathrm{ML}$ holding dam where impurities are removed. A controlled gravitational release allows the holding dam to empty in around two weeks. The usual policy has been to allow water to drain continually at the maximum rate. Subsequently, the water flows to a small wetland for further purification and is then supplied directly to consumers or pumped into an underground aquifer for longer term storage. Reclaimed water is used by local industry to replace more expensive mains water. The City of Salisbury supplies prescribed annual volumes to each of their clients. There is no guarantee that water will be available on any given day and no particular problem if the holding dam is emptied from time to time. Information about environmental policies and practices in the City of Salisbury can be found at www.salisbury.sa.gov.au/Our_City/Environment. For a full discussion of the Parafield system we refer to Piantadosi [16]. Piantadosi constructed a computer simulation of the system using Monte Carlo methods to generate realistic daily rainfall [18] and a pump-to-fill policy to move water from the capture dam to the holding dam. Piantadosi postulated that the pump-to-fill policy would minimise overflow and maximise the volume supplied to consumers. Piantadosi also described a modified Parafield system with constant demand in which water was pumped directly from the holding dam to consumers.

\subsection{A mathematical model for water storage in two connected dams}

We present a discrete state-space model of an urban water storage system consisting of two connected dams - a holding dam with capacity $m$ units and a capture dam with capacity $n$ units. We assume that $m \leq n$. A similar analysis applies for $m>n$. The state of the system is the content of the two dams. We assume stochastic supply and independent stochastic demand and use a pump-to-fill policy to transfer water from the capture dam to the holding dam. We also assume that pumping capacity is sufficient to ensure that the desired daily transfers are feasible. Our aim is to find new analytic formulae for the invariant state probabilities. Numerical calculation is not straightforward because the $(m+1)(n+1) \times(m+1)(n+1)$ transition matrix may be very large. We present a typical example with $m=n=50$. We use the terms supply and demand throughout but we may also think of inflow and outflow. Note that supply $=$ inflow + overflow and demand $=$ outflow + shortfall.

\subsection{More general models}

There are some factors we have not considered. In a realistic simulation water losses occur by evaporation and seepage. The simulation described in [19] has now been extended-as suggested in the concluding remarks - to include water loss at a rate given by an empirically defined seasonal constant multiplied by the exposed surface area. In a mathematical model such extensions may preclude analytic solutions and thereby obscure key insights. We have assumed that pumping capacity is sufficient to allow all desired daily transfers. This is certainly true in the Parafield system where the maximum pumping rate is $800 \mathrm{~L} / \mathrm{s}$. Nevertheless, development of a model with limited pumping capacity could be a worthwhile exercise. Correlation between supply and demand is likely in practice. We might expect demand to rise during dry spells when supply decreases. It may be possible to model correlation by considering joint probabilities for 
all possible transitions. It is difficult to allow seasonal variation in a theoretical model because the steady states are replaced by limit cycles. Alternatively, separate seasonal models could be used. See [7] for some examples.

\section{Previous research}

The mathematical literature on water storage in a single dam has developed largely from the work of Moran [10], [11] and his school. In particular, we cite Gani [5] and Yeo [20], [21]. For a system with two dams - a capture dam and a holding dam-a more complex model is required. For technical reasons we consider a discretised version of the problem which is formulated as a Markov decision process using a bivariate state space. The first state variable is the content of the holding dam-the level — and the second state variable is the content of the capture dam-the phase.

The pump-to-fill policy defines a Markov chain in which the transition matrix takes a characteristic regular block structure. For a system of two dams with stochastic supply and constant demand Howlett and Piantadosi [7] used Gaussian elimination (GE) and elementary matrix analytic methods (MAMs) to find elegant analytic formulae for the invariant level and phase probabilities. Although the derivations are complicated these formulae can be used conveniently in MATLAB ${ }^{\circledR}$ for numerical computations. It has been shown (see [14] and [15]) in quite general circumstances, that the pump-to-fill policy is optimal insofar as it minimises both the volume of overflow and the volume of shortfall. The proof is constructed by showing that the pump-to-fill policy is optimal for every possible event sequence. The MAMs developed in [8], [12], and [13] are well-suited to Markov chains with large transition matrices and the work in [6] and [9] is especially directed towards numerical calculations_-an important component of real-time management for a series of connected dams. In [17] MAMs and GE were used to calculate numerical values for invariant probabilities in a series of three dams-a capture dam, a holding dam, and a distribution dam-but the inherent algebraic complexity of the method has prevented any extension to larger systems.

Management of multiply-connected reservoirs has also been considered but the complexity of such systems generally precludes theoretical derivation of optimal solutions. Stochastic dynamic programming has been used to find effective suboptimal management policies. See [1], [2], and [3] for a comprehensive discussion.

\section{The main results}

The main results are developed for two connected dams with stochastic daily supply to the system and independent stochastic daily demand. A pump-to-fill policy is used to transfer water from the capture dam to the holding dam. Known results [7] are extended by exposing a much simpler intrinsic structure. If the holding dam has capacity $m$ units and the capture dam has capacity $n$ units then the Markov transition matrix is a large $(m+1)(n+1) \times(m+1)(n+1)$ matrix.

We consider a censored process by observing only transitions at the top level-when the holding dam is full-and show that the corresponding invariant probability can be expressed as a linear combination of shifted supply probability vectors. We show that the block transition matrix from any level other than the top level to any subsequent level is the product of a level-tolevel specific column vector and the supply probability row vector; see (5.2), below. Next, we show that invariant probabilities at each level can be calculated separately using only matrices of size $(n+1) \times(n+1)$ and that invariant probabilities for all levels other than the top level 
are scalar multiples of the supply probability vector. Numerical calculations are based on (5.1), (5.4), (5.9), and (5.10), below. We also outline an extension of the theory to a system of three dams

We present two examples for the modified Parafield system with stochastic supply $V$ where $\mathbb{E}[V]=2.099$. The first has stochastic demand $W$ with $\mathbb{E}[W]=2.000$ and the second has constant demand $d=2$. The invariant probabilities are very similar but the system with constant demand exhibits some characteristic oscillation in phase probabilities that are averaged out in the fully stochastic model. The main advantage of the stochastic model is that it is possible to set the average demand to a noninteger value and hence to design a balanced system.

\section{A system of two dams with constant demand}

We begin by describing a system of two dams with stochastic daily supply and constant daily demand. We use a discrete state-space model in which the holding dam has capacity $m$ and the capture dam has capacity $n$ where $m \leq n$. The system is managed using a pump-to-fill policy to transfer water from the capture dam to the holding dam. The system states are denoted by $S_{i j}=(i, j)$ where $i \in[0, m]$ is the content of the holding dam and $j \in[0, n]$ is the content of the capture dam. The states are ordered according to the following rules: $\left(i_{1}, j_{1}\right)$ precedes $\left(i_{2}, j_{2}\right)$ if $i_{1}<i_{2}$ or if $i_{1}=i_{2}$ and $j_{1}<j_{2}$. We write $\left(i_{1}, j_{1}\right) \prec\left(i_{2}, j_{2}\right)$. The state space

$$
S=\{(0,0), \ldots,(0, n) ;(1,0), \ldots,(1, n) ; \ldots ;(m, 0), \ldots,(m, n)\}
$$

contains $h=(m+1)(n+1)$ elements and can be expressed as $S=\bigcup_{i=0}^{m} L_{i}$ where the level $L_{i}$ is defined as the collection of all states $L_{i}=\{(i, 0),(i, 1), \ldots,(i, n)\}$. The first component (the content of the holding dam) refers to the level of the process and the second component (the content of the capture dam) refers to the phase.

\subsection{The pump-to-fill policy}

The following rules describe the state transitions for the pump-to-fill policy. We assume constant demand $d$ units per day from the holding dam where $d \geq 0$. The outflow from the holding dam is defined by $s=\min (d, i)$ where $i$ is the content of the holding dam. The supply is a random variable $V$. For each $r=0,1, \ldots$ the probability that $r$ units of water are supplied to the capture dam on any given day is denoted by $p_{r}=\mathbb{P}[V=r]$ and $p_{r}^{+}=\mathbb{P}[V \geq r]$ denotes the probability that at least $r$ units of water are supplied to the capture dam. The supply probability vector is

$$
\boldsymbol{p}=\left[p_{0}, p_{1}, \ldots, p_{n-1}, p_{n}^{+}\right] \in \mathbb{R}^{1 \times(n+1)} .
$$

We assume that $0<p_{0}<1$. The conditional probability that the following transitions will occur is the probability $p_{r}$ that $r$ units of water are supplied to the capture dam. Each day it is necessary to establish the order in which key actions are deemed to occur. Firstly, we pump water from the holding dam to users; secondly, we transfer water from the capture dam to the holding dam; thirdly, we receive the random supply to the capture dam. The transitions for the pump-to-fill policy are given here.

- For the state $(i, j)$ where $0 \leq i<d$ and $j \leq m$, pump $s=i$ units of water to users and pump all the water from the capture dam to the holding dam. If $r$ units of water enter the capture dam then $(i, j) \rightarrow(j, \min [r, n])$. If $r>n$ then the capture dam overflows and $r-n$ units of water are wasted.

- For the state $(i, j)$ where $0 \leq i<d$ and $j>m$, pump $s=i$ units of water to users and pump $m$ units from the capture dam. If $r$ units of water enter the capture dam then 
$(i, j) \rightarrow(m, \min [j-m+r, n])$. If $r>n+m-j$ then the capture dam overflows and $r-n-m+j$ units of water are wasted.

- For the state $(i, j)$ where $i \geq d$ and $j \leq m-i+d$, pump $s=d$ units of water to users and pump all the water from the capture dam to the holding dam. If $r$ units of water enter the capture dam then $(i, j) \rightarrow(i-d+j, \min [r, n])$. If $r>n$ then the capture dam overflows and $r-n$ units of water are wasted.

- For the state $(i, j)$ where $i \geq d$ and $j>m-i+d$, pump $s=d$ units of water to users and pump $m-i+d$ units of water to the holding dam. If $r$ units of water enter the capture dam then $(i, j) \rightarrow(m, \min [j-m+i-d+r, n])$. If $r>n+m-i+d-j$ then the capture dam overflows and $r-n-m+i-d+j$ units of water are wasted.

\subsection{The Markov model}

This model was proposed by Piantadosi [16] and is fully described in [7]. Define the simple substochastic matrix $\boldsymbol{A} \in \mathbb{R}^{(n+1) \times(n+1)}$ by

$$
\boldsymbol{A}=\left[\begin{array}{cccccc}
p_{0} & p_{1} & p_{2} & \cdots & p_{n-1} & p_{n}^{+} \\
0 & 0 & 0 & \cdots & 0 & 0 \\
\vdots & \vdots & \vdots & & \vdots & \vdots \\
0 & 0 & 0 & \cdots & 0 & 0
\end{array}\right]
$$

and substochastic matrices $\boldsymbol{A}_{\boldsymbol{i}} \in \mathbb{R}^{(n+1) \times(n+1)}$ for each $i=0, \ldots, m$ in which the first $i$ rows are zero in the general form

$$
\boldsymbol{A}_{i}=\left[\begin{array}{ccccccc}
0 & 0 & \cdots & 0 & \cdots & 0 & 0 \\
\vdots & \vdots & & \vdots & & \vdots & \vdots \\
0 & 0 & \cdots & 0 & \cdots & 0 & 0 \\
p_{0} & p_{1} & \cdots & p_{n-i} & \cdots & p_{n-1} & p_{n}^{+} \\
0 & p_{0} & \cdots & p_{n-i-1} & \cdots & p_{n-2} & p_{n-1}^{+} \\
\vdots & \vdots & & \vdots & & \vdots & \vdots \\
0 & 0 & \cdots & p_{0} & \cdots & p_{i-1} & p_{i}^{+}
\end{array}\right]
$$

If we define the permutation matrix $\Sigma \in \mathbb{R}^{(n+1) \times(n+1)}$ by

$$
\boldsymbol{\Sigma}=\left[\begin{array}{cccccc}
0 & 0 & 0 & \cdots & 0 & 1 \\
1 & 0 & 0 & \cdots & 0 & 0 \\
0 & 1 & 0 & \cdots & 0 & 0 \\
\vdots & \vdots & \vdots & & \vdots & \vdots \\
0 & 0 & 0 & \cdots & 1 & 0
\end{array}\right]
$$

and if $m \leq n$ then the transition matrix for the system is given by

$$
\boldsymbol{Q}_{d}=\left[\begin{array}{ccccccc}
\boldsymbol{A} & \Sigma \boldsymbol{A} & \cdots & \Sigma^{m-d+1} \boldsymbol{A} & \cdots & \Sigma^{m-1} \boldsymbol{A} & \boldsymbol{A}_{m} \\
\vdots & \vdots & & \vdots & & \vdots & \vdots \\
\boldsymbol{A} & \Sigma \boldsymbol{A} & \cdots & \Sigma^{m-d+1} \boldsymbol{A} & \cdots & \Sigma^{m-1} \boldsymbol{A} & \boldsymbol{A}_{m} \\
0 & \boldsymbol{A} & \cdots & \Sigma^{m-d} \boldsymbol{A} & \cdots & \Sigma^{m-2} \boldsymbol{A} & \boldsymbol{A}_{m-1} \\
\vdots & \vdots & & \vdots & & \vdots & \vdots \\
0 & 0 & \cdots & \boldsymbol{A} & \cdots & \Sigma^{d-1} \boldsymbol{A} & \boldsymbol{A}_{d}
\end{array}\right]=\left[\begin{array}{c}
\boldsymbol{Q}_{d, 0} \\
\vdots \\
\boldsymbol{Q}_{d, d} \\
\boldsymbol{Q}_{d, d+1} \\
\vdots \\
\boldsymbol{Q}_{d, m}
\end{array}\right] \in \mathbb{R}^{h \times h}
$$


If the vector

$$
\boldsymbol{x}_{d}^{(t)}=\left[\boldsymbol{x}_{d, 0}^{(t)}, \boldsymbol{x}_{d, 1}^{(t)}, \ldots, \boldsymbol{x}_{d, m}^{(t)}\right] \in \mathbb{R}^{1 \times h}
$$

denotes the probable state of the system at time $t$, where $\boldsymbol{x}_{d, i}^{(t)} \in \mathbb{R}^{1 \times(n+1)}$ is the probability that the state is in level $i$, then evolution of the system is defined by

$$
\boldsymbol{x}_{d}^{(t)} \boldsymbol{Q}_{d}=\boldsymbol{x}_{d}^{(t+1)} \quad \Longleftrightarrow \quad \boldsymbol{x}_{d, 0}^{(t)} \boldsymbol{Q}_{d, 0}+\boldsymbol{x}_{d, 1}^{(t)} \boldsymbol{Q}_{d, 1}+\cdots+\boldsymbol{x}_{d, m}^{(t)} \boldsymbol{Q}_{d, m}=\boldsymbol{x}_{d}^{(t+1)} .
$$

If we denote the invariant probability by

$$
\boldsymbol{\pi}_{d}=\left[\boldsymbol{\pi}_{d, 0}, \boldsymbol{\pi}_{d, 1}, \cdots, \boldsymbol{\pi}_{d, m-1}, \boldsymbol{\pi}_{d, m}\right] \in \mathbb{R}^{1 \times h},
$$

where the vector component $\boldsymbol{\pi}_{d, i} \in \mathbb{R}^{1 \times(n+1)}$ for each $i=0,1, \ldots, m$ denotes the invariant probability for level $i$, then the invariant probability satisfies the equation

$$
\boldsymbol{\pi}_{d} \boldsymbol{Q}_{d}=\boldsymbol{\pi}_{d} \quad \Longleftrightarrow \quad \boldsymbol{\pi}_{d, 0} \boldsymbol{Q}_{d, 0}+\boldsymbol{\pi}_{d, 1} \boldsymbol{Q}_{d, 1}+\cdots+\boldsymbol{\pi}_{d, m} \boldsymbol{Q}_{d, m}=\boldsymbol{\pi}_{d} .
$$

\section{A system of two dams with independent stochastic demand}

We extend the basic model with constant demand to a model with stochastic demand defined by a probability vector

$$
\boldsymbol{q}=\left[q_{0}, q_{1}, \ldots, q_{m-1}, q_{m}^{+}\right] \in \mathbb{R}^{1 \times(m+1)},
$$

where $q_{s}$ denotes the probability of demand $d=s$ for each $s=0,1, \ldots$ and $q_{s}^{+}$denotes the probability of demand $d \geq s$. The model with stochastic demand is a linear combination of the models with constant demand, and so the transition matrix is

$$
\boldsymbol{T}=q_{0} \boldsymbol{Q}_{0}+q_{1} \boldsymbol{Q}_{1}+\cdots+q_{m-1} \boldsymbol{Q}_{m-1}+q_{m}^{+} \boldsymbol{Q}_{m} \in \mathbb{R}^{h \times h} .
$$

To emphasise the general structure of this transition matrix we introduce some new notation. If we write $\boldsymbol{T}=\left[\boldsymbol{T}_{i, j}\right]$ where $\boldsymbol{T}_{i, j} \in \mathbb{R}^{(n+1) \times(n+1)}$ then we have

$$
\boldsymbol{T}_{i, j}= \begin{cases}q_{i}^{+} \Sigma^{j} \boldsymbol{A}+\sum_{k=1}^{\min (i, j)} q_{i-k} \Sigma^{j-k} \boldsymbol{A} & \text { for } 0 \leq j<m, \\ q_{i}^{+} \boldsymbol{A}_{m}+\sum_{k=1}^{i} q_{i-k} \boldsymbol{A}_{m-k} & \text { for } j=m,\end{cases}
$$

and $0 \leq i \leq m$. Hence, if we define matrices $\Phi_{i, j} \in \mathbb{R}^{(n+1) \times(n+1)}$ by the formulae

$$
\Phi_{i, j}= \begin{cases}q_{i}^{+} \Sigma^{j}+\sum_{k=1}^{\min (i, j)} q_{i-k} \Sigma^{j-k} & \text { for } 0 \leq j<m, \\ q_{i}^{+} \boldsymbol{A}_{m}+\sum_{k=1}^{i} q_{i-k} \boldsymbol{A}_{m-k} & \text { for } j=m,\end{cases}
$$

and $0 \leq i \leq m$, then we can write

$$
\boldsymbol{T}=\left[\begin{array}{ccccc}
\Phi_{0,0} \boldsymbol{A} & \Phi_{0,1} \boldsymbol{A} & \ldots & \Phi_{0, m-1} \boldsymbol{A} & \Phi_{0, m} \\
\Phi_{1,0} \boldsymbol{A} & \Phi_{1,1} \boldsymbol{A} & \ldots & \Phi_{1, m-1} \boldsymbol{A} & \Phi_{1, m} \\
\vdots & \vdots & \ddots & \vdots & \vdots \\
\Phi_{m, 0} \boldsymbol{A} & \Phi_{m, 1} \boldsymbol{A} & \ldots & \Phi_{m, m-1} \boldsymbol{A} & \Phi_{m, m}
\end{array}\right] .
$$


If we denote the invariant probability by

$$
\boldsymbol{\pi}=\left[\boldsymbol{\pi}_{0}, \boldsymbol{\pi}_{1}, \boldsymbol{\pi}_{2}, \cdots \boldsymbol{\pi}_{m-1}, \boldsymbol{\pi}_{m}\right] \in \mathbb{R}^{1 \times h},
$$

where the vector component $\pi_{i} \in \mathbb{R}^{1 \times(n+1)}$ for each $i=0,1, \ldots, m$ denotes the invariant probability for level $i$, then the invariant probability satisfies the equation

$$
\begin{aligned}
& \boldsymbol{\pi} \boldsymbol{T}=\boldsymbol{\pi} \quad \Longleftrightarrow \quad \boldsymbol{\pi}_{0} \Phi_{0, j} \boldsymbol{A}+\cdots+\boldsymbol{\pi}_{m} \Phi_{m, j} \boldsymbol{A}=\boldsymbol{\pi}_{j} \quad(\text { for } j=0, \ldots, m-1) \\
& \text { and } \quad \pi_{0} \Phi_{0, m}+\cdots+\pi_{m} \Phi_{m, m}=\pi_{m} \quad(\text { for } j=m) .
\end{aligned}
$$

\subsection{The censored Markov process}

We partition $\boldsymbol{T}$ as

$$
\boldsymbol{T}=\left[\begin{array}{cc}
\boldsymbol{T}_{1} & \boldsymbol{T}_{2} \\
\boldsymbol{T}_{3} & \Phi_{m, m}
\end{array}\right],
$$

where $\boldsymbol{T}_{1} \in \mathbb{R}^{m(n+1) \times m(n+1)}, \boldsymbol{T}_{2} \in \mathbb{R}^{m(n+1) \times(n+1)}$, and $\boldsymbol{T}_{3} \in \mathbb{R}^{(n+1) \times m(n+1)}$ and censor the process by observing it only when the state lies in level $m$. The censored process has transition matrix

$$
\boldsymbol{S}=\Phi_{m, m}+\boldsymbol{T}_{3}\left(\boldsymbol{I}-\boldsymbol{T}_{1}\right)^{-1} \boldsymbol{T}_{2} \in \mathbb{R}^{(n+1) \times(n+1)},
$$

and is irreducible with invariant probability $\rho=\left[\rho_{0}, \rho_{1}, \ldots, \rho_{n}\right] \in \mathbb{R}^{1 \times(n+1)}$ satisfying the equation

$$
\rho S=\rho .
$$

Furthermore, since $\rho$ is the relative invariant probability of the phases given that the level is $m$, it follows that $\pi_{m}=\alpha_{m} \rho$, where the constant $\alpha_{m} \in[0,1]$ is the invariant probability for level $m$. Note that we can represent $\rho$ in terms of shifted supply probability vectors as follows. Since $p_{0}>0$, the vectors

$$
\delta^{k} \boldsymbol{p}=\left[0,0, \ldots, 0, p_{0}, p_{1}, \ldots, p_{n-k}^{+}\right],
$$

for $k=0,1, \ldots, n$, are a basis for $\mathbb{R}^{1 \times(n+1)}$. Thus, we can write $\boldsymbol{\rho}=\sum_{k=0}^{n} \gamma_{k} \delta^{k} \boldsymbol{p}$ for suitably chosen constants $\gamma_{k} \in \mathbb{R}$. Note that $\delta^{0} \boldsymbol{p}=\boldsymbol{p}$.

\subsection{The key simplification}

For $0 \leq i \leq n$ let $\boldsymbol{e}_{i} \in \mathbb{R}^{(n+1) \times 1}$ denote the unit column vector with 1 in the $i$ th position and 0 elsewhere. Now, $\Sigma^{k} A=\boldsymbol{e}_{k} \boldsymbol{p}$ for $0 \leq k \leq n$; hence,

$$
\Phi_{i, j} \boldsymbol{A}=\boldsymbol{u}_{i, j} \boldsymbol{p}
$$

where $\boldsymbol{u}_{i, j} \in \mathbb{R}^{(n+1) \times 1}$ and $\boldsymbol{u}_{i, j} \geq \mathbf{0}$ for each $0 \leq i \leq m$ and $0 \leq j<m-1$. Hence, we can simplify the expressions for the two key block matrices $\boldsymbol{T}_{1}$ and $\overline{\boldsymbol{T}}_{3}$ by writing

$$
\boldsymbol{T}_{1}=\left[\boldsymbol{u}_{i, j} \boldsymbol{p}\right], \quad \boldsymbol{T}_{3}=\left[\boldsymbol{u}_{m, j} \boldsymbol{p}\right] .
$$

It is now easy to show that powers of $\boldsymbol{T}_{1}$ also take a simple form. Indeed, we can write

$$
\boldsymbol{T}_{1}^{S}=\left[\boldsymbol{u}_{i, j}^{(s)} \boldsymbol{p}\right],
$$

for each $s \in \mathbb{N}$, where $\boldsymbol{u}_{i, j}^{(s)} \in \mathbb{R}^{(n+1) \times 1}$ and $\boldsymbol{u}_{i, j}^{(s)} \geq \mathbf{0}$ for each $0 \leq i \leq m-1$ and $0 \leq j \leq m-1$. Hence, $\boldsymbol{B}=\sum_{s=1}^{\infty} T_{1}^{s} \in \mathbb{R}^{m(n+1) \times m(n+1)}$ can be written in the form

$$
\boldsymbol{B}=\left[\boldsymbol{v}_{i, j} p\right],
$$

where $\boldsymbol{v}_{i, j} \in \mathbb{R}^{(n+1) \times 1}$ and $\boldsymbol{v}_{i, j} \geq \mathbf{0}$ for each $0 \leq i \leq m-1$ and $0 \leq j \leq m-1$. The matrix $\boldsymbol{B}$ satisfies the identity

$$
\boldsymbol{I}+\boldsymbol{B}=\left(\boldsymbol{I}-\boldsymbol{T}_{1}\right)^{-1} .
$$




\subsection{The reduction process}

It is convenient to rearrange the matrix multiplications. To do this, define nonnegative elements

$$
\theta_{i, j}=\boldsymbol{p} \boldsymbol{u}_{i, j} \in \mathbb{R}, \quad \omega_{i, j}=\boldsymbol{p} \boldsymbol{v}_{i, j} \in \mathbb{R},
$$

for each $0 \leq i \leq m-1$ and $0 \leq j \leq m-1$; hence, define new matrices

$$
\boldsymbol{\Theta}=\left[\boldsymbol{p} \boldsymbol{u}_{i, j}\right] \in \mathbb{R}^{m \times m}, \quad \boldsymbol{\Omega}=\left[\boldsymbol{p} \boldsymbol{v}_{i, j}\right] \in \mathbb{R}^{m \times m} .
$$

It is a matter of elementary matrix multiplication to show that

$$
\boldsymbol{\Theta}^{s}=\left[\boldsymbol{p} \boldsymbol{u}_{i, j}^{(s)}\right],
$$

where $\boldsymbol{u}_{i, j}^{(s)}$ is defined earlier in (5.3) for $0 \leq i \leq m-1$ and $0 \leq j \leq m-1$. Hence, we deduce that

$$
\boldsymbol{\Omega}=\sum_{s=1}^{\infty} \boldsymbol{\Theta}^{s} \quad \Longleftrightarrow \quad \boldsymbol{I}+\boldsymbol{\Omega}=(\boldsymbol{I}-\boldsymbol{\Theta})^{-1} .
$$

The relationship between $\boldsymbol{\Omega}$ and $\boldsymbol{\Theta}$ is equivalent to the relationship between $\boldsymbol{B}$ and $\boldsymbol{T}$ and essentially involves a simple rearrangement of the same matrix multiplications.

\subsection{The solution}

We can now begin the solution procedure. From the equation $\pi=\pi \boldsymbol{T}$ we have

$$
\boldsymbol{\pi}_{i}=\alpha_{i} \boldsymbol{p}
$$

where

$$
\alpha_{i}=\sum_{k=0}^{m} \boldsymbol{\pi}_{k} \boldsymbol{u}_{k, i}
$$

is the invariant probability for level $i$ for each $0 \leq i<m$. Since $\boldsymbol{\pi}_{k} \boldsymbol{u}_{k, i}=\alpha_{k} \boldsymbol{p} \boldsymbol{u}_{k, i}=\alpha_{k} \theta_{k, i}$ for $0 \leq k \leq m-1$ and since $\boldsymbol{\pi}_{m}=\alpha_{m} \boldsymbol{\rho}$, this becomes

$$
\alpha_{i}=\sum_{k=0}^{m-1} \alpha_{k} \theta_{k, i}+\alpha_{m} \tau_{i}
$$

where we have defined $\tau_{i}=\boldsymbol{\rho} \boldsymbol{u}_{m, i} \in \mathbb{R}$ for each $0 \leq i<m$. We use the vector notation

$$
\boldsymbol{\alpha}=\left[\alpha_{0}, \ldots, \alpha_{m-1}\right] \in \mathbb{R}^{1 \times m}, \quad \boldsymbol{\tau}=\left[\tau_{0}, \ldots, \tau_{m-1}\right] \in \mathbb{R}^{1 \times m}
$$

to collectively denote these terms. Now we can write (5.5) more compactly in the form

$$
\boldsymbol{\alpha}=\boldsymbol{\alpha} \boldsymbol{\Theta}+\alpha_{m} \boldsymbol{\tau} \quad \Longleftrightarrow \quad \boldsymbol{\alpha}=\alpha_{m} \boldsymbol{\tau}(\boldsymbol{I}-\boldsymbol{\Theta})^{-1} .
$$

If $\boldsymbol{f}_{i} \in \mathbb{R}^{m \times 1}$ is defined for $0 \leq i \leq m-1$ as the unit column vector with 1 in the $i$ th position and 0 elsewhere then it follows from (5.6) that the probability for level $i$ is given by

$$
\alpha_{i}=\boldsymbol{\alpha} \boldsymbol{f}_{i}=\alpha_{m} \boldsymbol{\tau}(\boldsymbol{I}-\boldsymbol{\Theta})^{-1} \boldsymbol{f}_{i},
$$

for $0 \leq i \leq m-1$. Since the total probability for all levels is 1 we have

$$
\sum_{i=0}^{m-1} \alpha_{m} \boldsymbol{\tau}(\boldsymbol{I}-\boldsymbol{\Theta})^{-1} \boldsymbol{f}_{i}+\alpha_{m}=1 \quad \Longleftrightarrow \quad \alpha_{m}=\frac{1}{1+\boldsymbol{\tau}(\boldsymbol{I}-\boldsymbol{\Theta})^{-1} \sum_{i=0}^{m-1} \boldsymbol{f}_{i}} .
$$


If we define $f=\sum_{i=0}^{m-1} \boldsymbol{f}_{i} \in \mathbb{R}^{m \times 1}$ as the vector with 1 in every position then the previous expression can be rewritten as

$$
\alpha_{m}=\frac{1}{1+\boldsymbol{\tau}(\boldsymbol{I}-\boldsymbol{\Theta})^{-1} \boldsymbol{f}} .
$$

For each $0 \leq i<m$ it follows from (5.7) and (5.8) that

$$
\boldsymbol{\pi}_{i}=\alpha_{i} \boldsymbol{p}=\alpha_{m} \boldsymbol{\tau}(\boldsymbol{I}-\boldsymbol{\Theta})^{-1} \boldsymbol{f}_{i} \boldsymbol{p}=\frac{\boldsymbol{\tau}(\boldsymbol{I}-\boldsymbol{\Theta})^{-1} \boldsymbol{f}_{i} \boldsymbol{p}}{1+\boldsymbol{\tau}(\boldsymbol{I}-\boldsymbol{\Theta})^{-1} \boldsymbol{f}} .
$$

Of course, we have already established that

$$
\boldsymbol{\pi}_{m}=\frac{\rho}{1+\boldsymbol{\tau}(\boldsymbol{I}-\boldsymbol{\Theta})^{-1} \boldsymbol{f}} .
$$

\section{Examples}

\subsection{Example 1: Stochastic supply and independent stochastic demand}

The rainfall data at Parafield has been collected over an approximate 100 year time-frame. Observations of the Parafield system show that supply is directly proportional to rainfall with $1 \mathrm{~mm}$ of rainfall generating approximately $1.75 \mathrm{ML}$ of inflow. In this example we assume a stochastic daily supply $V$ defined by a discrete approximation to the cumulative distribution function

$$
F[v]=p_{0}+\left(1-p_{0}\right) \int_{0}^{v} x^{k-1} \mathrm{e}^{-x / \theta} \mathrm{d} x,
$$

where the parameters $p_{0}, k$, and $\theta$ were determined by the method of maximum likelihood from the supply generated by observed rainfall over the past 100 years on October 15 . The supply probability $\boldsymbol{p} \in \mathbb{R}^{1 \times 51}$ is given approximately by

$$
\begin{aligned}
\boldsymbol{p} \approx & {[0.7114,0.0491,0.0348,0.0284,0.0238,0.0202,0.0173,0.0149,0.0129,} \\
& 0.0111,0.0097,0.0084,0.0073,0.0063,0.0055,0.0048,0.0042,0.0037, \\
& 0.0032,0.0028,0.0025,0.0022,0.0019,0.0016,0.0014,0.0013,0.0011, \\
& 0.0010,0.0009,0.0007,0.0007,0.0006,0.0005,0.0004,0.0004,0.0003, \\
& 0.0003,0.0003,0.0002,0.0002,0.0002,0.0002,0.0001,0.0001,0.0001, \\
& 0.0001,0.0001,0.0001,0.0001,0.0001,0.0004]
\end{aligned}
$$

We refer to [7] for further details. In this example the stochastic demand $W$ is generated by the binomial probability vector $\boldsymbol{q} \in \mathbb{R}^{1 \times 51}$ given by

$$
\boldsymbol{q}=\left[\begin{array}{lllllllll}
0.0778 & 0.2592 & 0.3456 & 0.2304 & 0.0768 & 0.0102 & 0 & \cdots & 0
\end{array}\right],
$$

where $q_{w}=\left(\begin{array}{c}5 \\ w\end{array}\right)(0.4)^{w}(0.6)^{5-w}$ for $w=0, \ldots, 5$. In practice, demand probabilities could be constructed by observation of daily demand. We might expect that the volume quotas promised to consumers would be randomly accessed on a daily basis but fully used over the relevant time-period. A well-designed system should be approximately balanced. That is, the expected demand should be approximately equal to the expected supply. In this example the expected daily supply is $\mathbb{E}[V] \approx 2.099 \mathrm{ML}$ and the expected daily demand is $\mathbb{E}[W]=2.000 \mathrm{ML}$. The supply and demand probabilities are shown in Figure 1. 

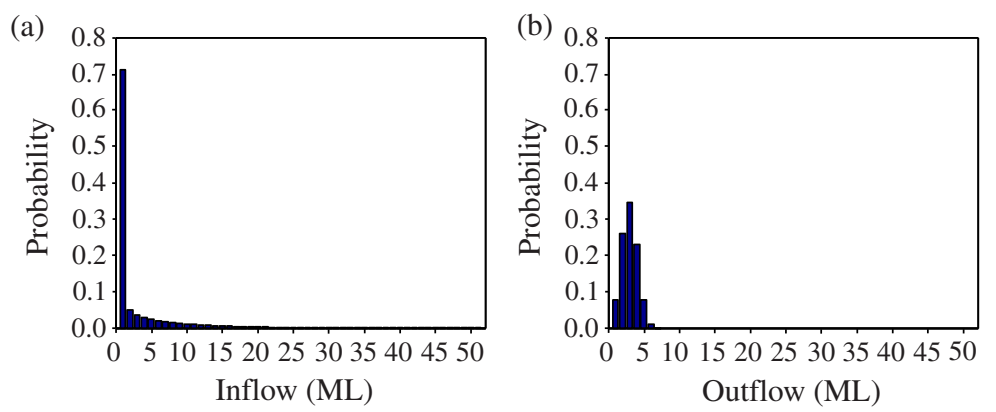

FIGURE 1: The supply probabilities (part (a)) and the demand probabilities (part (b)) from Example 1.
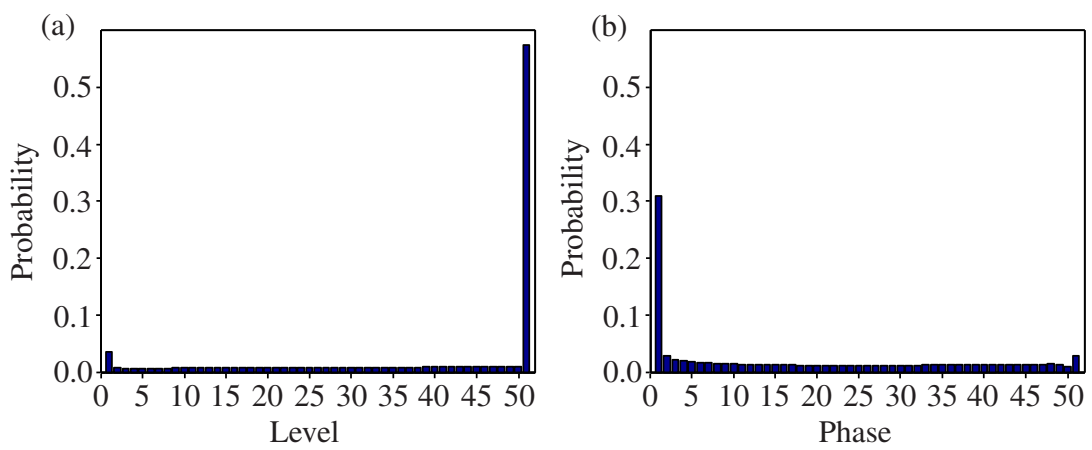

FIGURE 2: The level probabilities (part (a)) and the phase probabilities (part (b)) from Example 1.

The vector $\lambda=\left[\boldsymbol{\alpha}, \alpha_{50}\right]$ is the vector of invariant probabilities $\lambda_{i}=\alpha_{i} \in[0,1]$ for each level $i$ with $0 \leq i \leq 50$. The component $\lambda_{i}$ is the probability that the holding dam will contain $i$ units of water. We may define the vector $\boldsymbol{\mu}=\left[\mu_{0}, \mu_{1}, \ldots, \mu_{50}\right]$ of invariant probabilities $\mu_{j} \in[0,1]$ for each phase $j$ with $0 \leq j \leq 50$ by setting

$$
\boldsymbol{\mu}=\sum_{i=0}^{50} \boldsymbol{\pi}_{i}=\sum_{i=0}^{49} \lambda_{i} \boldsymbol{p}+\lambda_{50} \boldsymbol{\rho}=\left(1-\lambda_{50}\right) \boldsymbol{p}+\lambda_{50} \boldsymbol{\rho} .
$$

The component $\mu_{j}$ is the probability that the capture dam will contain $j$ units of water. The vector $\rho \in \mathbb{R}^{1 \times 51}$ defines the relative phase probabilities at level 50. In other words, $\rho$ shows the steady state probabilities for the content of the capture dam when the holding dam is full. The steady state level and phase probabilities are shown in Figure 2. The relative phase probabilities at level 50 are shown in Figure 3.

The detailed calculations show that

$$
\begin{aligned}
\lambda \approx & {[0.0350,0.0073,0.0069,0.0069,0.0068,0.0068,0.0069,0.0069,0.0069,} \\
& 0.0070,0.0070,0.0071,0.0071,0.0072,0.0072,0.0073,0.0073,0.0074, \\
& 0.0075,0.0075,0.0076,0.0076,0.0077,0.0077,0.0078,0.0079,0.0079, \\
& 0.0080,0.0081,0.0081,0.0082,0.0082,0.0083,0.0084,0.0084,0.0085, \\
& 0.0086,0.0086,0.0087,0.0088,0.0088,0.0089,0.0090,0.0090,0.0091, \\
& 0.0092,0.0093,0.0093,0.0094,0.0095,0.5752],
\end{aligned}
$$




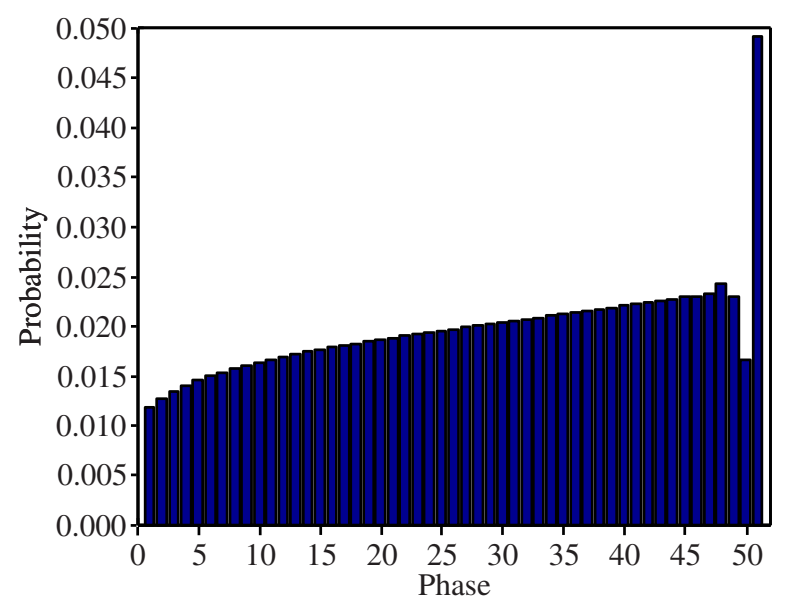

FIGURE 3: The relative phase probabilities at level 50 from Example 1.

$$
\begin{aligned}
\mu \approx & {[0.3090,0.0282,0.0225,0.0201,0.0184,0.0172,0.0162,0.0153,0.0147,} \\
& 0.0141,0.0137,0.0133,0.0129,0.0127,0.0125,0.0123,0.0121,0.0120, \\
& 0.0119,0.0119,0.0118,0.0118,0.0118,0.0118,0.0118,0.0118,0.0119, \\
& 0.0119,0.0119,0.0120,0.0121,0.0121,0.0122,0.0122,0.0123,0.0124, \\
& 0.0125,0.0126,0.0126,0.0127,0.0128,0.0129,0.0130,0.0131,0.0132, \\
& 0.0132,0.0134,0.0139,0.0132,0.0095,0.0284],
\end{aligned}
$$

and

$$
\begin{aligned}
\rho \approx & {[0.0118,0.0127,0.0134,0.0140,0.0145,0.0149,0.0153,0.0157,0.0160,} \\
& 0.0163,0.0166,0.0169,0.0171,0.0174,0.0176,0.0178,0.0180,0.0182, \\
& 0.0184,0.0186,0.0188,0.0189,0.0191,0.0193,0.0195,0.0196,0.0198, \\
& 0.0200,0.0201,0.0203,0.0205,0.0206,0.0208,0.0210,0.0211,0.0213, \\
& 0.0215,0.0216,0.0218,0.0220,0.0222,0.0223,0.0225,0.0227,0.0229, \\
& 0.0229,0.0232,0.0242,0.0230,0.0165,0.0490] .
\end{aligned}
$$

The probability that the holding dam will be full is $\lambda_{50} \approx 0.5752$ while the probability that the holding dam will be empty is $\lambda_{0} \approx 0.0350$.

\subsection{Example 2: Stochastic supply and constant demand}

For comparison, we have also calculated steady state probabilities for an identical system with the same supply probabilities but with constant demand $d=2$. The case $d=2$ is obtained by setting $\boldsymbol{q}=[0,0,1,0, \ldots]$. The level and phase probabilities are shown in Figure 4.

The probability that the holding dam will be full is $\lambda_{50} \approx 0.5782$ while the probability that the holding dam will be empty is $\lambda_{0} \approx 0.0345$. These probabilities are almost the same as the corresponding probabilities for the fully stochastic system. Since the demand is slightly less than the expected supply it is a little more likely that the holding dam will be full than would be the case in a perfectly balanced system. 

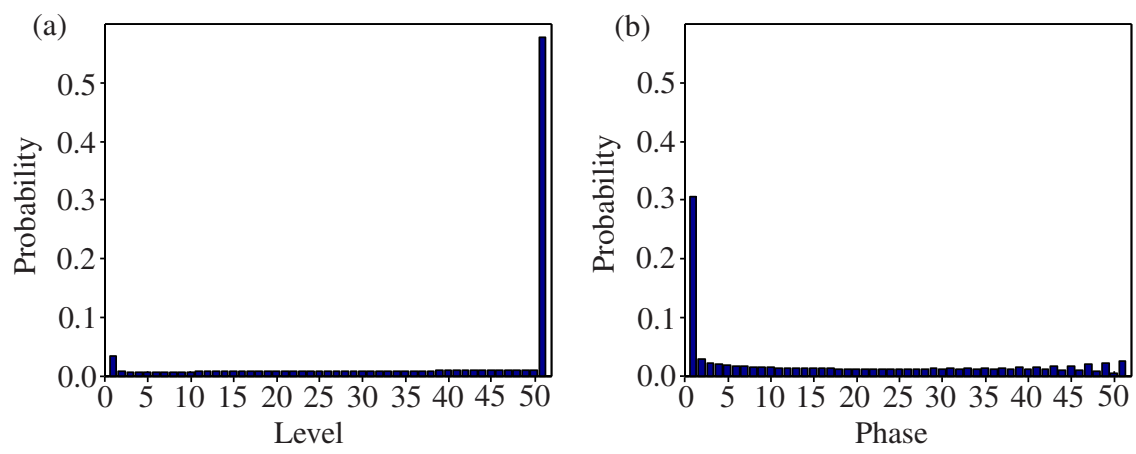

FIgURE 4: The level probabilities for $d=2$ (part (a)) and the phase probabilities for $d=2$ (part (b)) for Example 2.

The detailed calculations show that

$$
\begin{aligned}
\lambda \approx[ & 0.0345,0.0072,0.0068,0.0067,0.0067,0.0067,0.0068,0.0068,0.0068, \\
& 0.0069,0.0069,0.0070,0.0070,0.0071,0.0072,0.0072,0.0073,0.0073, \\
& 0.0074,0.0074,0.0075,0.0076,0.0076,0.0077,0.0078,0.0078,0.0079, \\
& 0.0079,0.0080,0.0081,0.0081,0.0082,0.0083,0.0083,0.0084,0.0085, \\
& 0.0085,0.0086,0.0087,0.0088,0.0088,0.0089,0.0090,0.0090,0.0091, \\
& 0.0092,0.0093,0.0093,0.0094,0.0095,0.5782]
\end{aligned}
$$

and

$$
\begin{aligned}
\boldsymbol{\mu} \approx & {[0.3069,0.0281,0.0224,0.0201,0.0184,0.0172,0.0162,} \\
& 0.0153,0.0147,0.0141,0.0137,0.0133,0.0130,0.0127,0.0125,0.0123, \\
& 0.0122,0.0121,0.0121,0.0119,0.0120,0.0119,0.0120,0.0118,0.0120, \\
& 0.0119,0.0121,0.0119,0.0123,0.0119,0.0125,0.0119,0.0127,0.0119, \\
& 0.0131,0.0119,0.0135,0.0118,0.0141,0.0115,0.0148,0.0110,0.0158, \\
& 0.0102,0.0172,0.0090,0.0191,0.0071,0.0219,0.0043,0.0259]
\end{aligned}
$$

Note that $\mu_{50}, \mu_{48}, \mu_{46}, \ldots$ are significantly greater than the intermediate terms $\mu_{49}, \mu_{47}$, $\mu_{45}, \ldots$ in this example. Since

$$
\boldsymbol{\mu}=\left(1-\lambda_{50}\right) \boldsymbol{p}+\lambda_{50} \rho
$$

and since $p_{j-1}>p_{j}$ for all $j \in \mathbb{N}$, it follows that the oscillations in $\mu$ must come from the oscillations in $\rho$. These oscillations can be seen clearly in Figure 5. The oscillations may be explained as follows. The vector $\rho$ describes the conditional probabilities for the content of the capture dam when the holding dam is full. If the capture dam is also full and there is no supply for the next few days - a reasonably likely event—-then the content of the capture dam will fall by two units each day. Because $\rho_{50}$ is relatively large we might expect heightened values for $\rho_{48}, \rho_{46}, \rho_{44}, \ldots$, and so on.

The fully stochastic system in Example 1 has expected demand $\mathbb{E}[W]=2$ and hence we would expect the steady state probabilities to be similar. This is indeed the case. Note, however, that the relative phase probabilities at level 50 are smoother in the stochastic system because the outflow is obtained as an average of different outflows. 


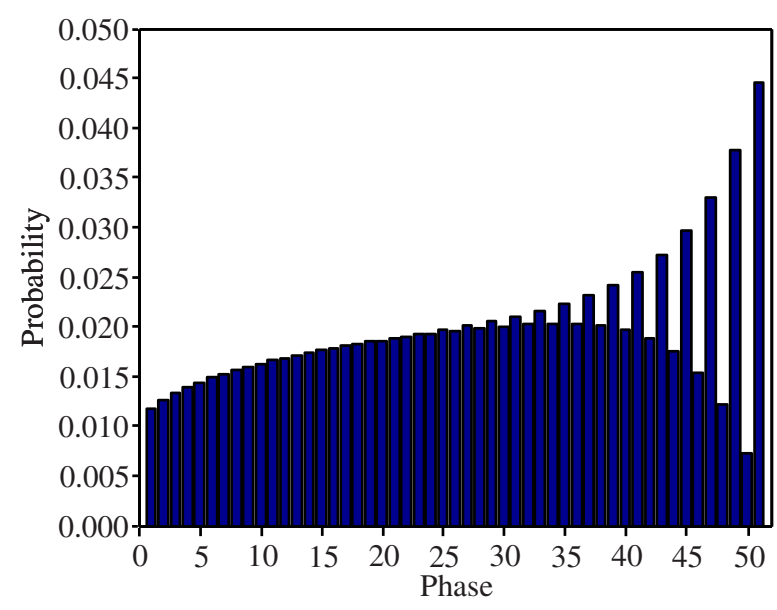

FIGURE 5: The relative phase probabilities at level 50 for $d=2$ for Example 2.

\section{Numerical calculations}

The transition matrix $\boldsymbol{T} \in \mathbb{R}^{h \times h}$ may be very large. In our examples we have $h=2601$. Consequently, it is desirable to make the numerical calculations as efficient as possible. We used MATLAB for all of our numerical calculations. There is no particular problem in constructing large matrices and storing them in MATLAB but it is prudent to avoid, where possible, complex calculations with these large matrices.

\subsection{The new calculations}

Rather than find the steady state for $\boldsymbol{T} \in \mathbb{R}^{2601 \times 2601}$ directly we find it indirectly by first calculating the steady state for $\boldsymbol{S} \in \mathbb{R}^{51 \times 51}$. However, $\boldsymbol{S}=\varphi_{m m}+\boldsymbol{T}_{3}\left(\boldsymbol{I}-\boldsymbol{T}_{1}\right)^{-1} \boldsymbol{T}_{2}$, and it would be fair to observe that $\boldsymbol{T}_{1} \in \mathbb{R}^{2551 \times 2551}$. How then do we avoid complex calculations to determine $\left(\boldsymbol{I}-\boldsymbol{T}_{1}\right)^{-1}$ ? We may write

$$
\boldsymbol{T}_{1}=\left[\boldsymbol{\Psi}_{i, j}\right], \quad \text { where } \boldsymbol{\Psi}_{i, j}=\boldsymbol{u}_{i, j} \boldsymbol{p} \in \mathbb{R}^{51 \times 51},
$$

and

$$
\boldsymbol{u}_{i, j}=q_{i}^{+} \boldsymbol{e}_{j}+\sum_{k=1}^{\min (i, j)} q_{i-k} \boldsymbol{e}_{j-k} \in \mathbb{R}^{51 \times 1},
$$

for each $i=0, \ldots, 49$ and $j=0, \ldots, 49$. We will use the matrix $\boldsymbol{\Theta}=\left[\theta_{i, j}\right] \in \mathbb{R}^{50 \times 50}$ in our calculations. Note that $\theta_{i, j}=\boldsymbol{p} \boldsymbol{u}_{i, j} \in \mathbb{R}$ for each $i=0, \ldots, 49$ and $j=0, \ldots, 49$. If we write $\boldsymbol{T}_{1}^{r}=\left[\boldsymbol{\Psi}_{i, j}^{(r)}\right]$ and $\boldsymbol{\Theta}^{s}=\left[\theta_{i, j}^{(s)}\right]$ then we wish to show that

$$
\boldsymbol{\Psi}_{i, j}^{(r)}=\sum_{k=0}^{49} \boldsymbol{u}_{i, k} \theta_{k, j}^{(r-1)} \boldsymbol{p}
$$

for each $r \in \mathbb{N}$. Indeed, a simple inductive argument shows us that

$$
\boldsymbol{\Psi}_{i, j}^{(r+1)}=\sum_{h=0}^{49} \boldsymbol{u}_{i, h} \boldsymbol{p} \sum_{k=0}^{49} \boldsymbol{u}_{h, k} \theta_{k, j}^{(r-1)} \boldsymbol{p}=\sum_{h=0}^{49} \boldsymbol{u}_{i, h} \sum_{k=0}^{49} \theta_{h, k} \theta_{k, j}^{(r-1)} \boldsymbol{p}=\sum_{h=0}^{49} \boldsymbol{u}_{i, h} \theta_{h, j}^{(r)} \boldsymbol{p},
$$


as required. By summing over $r$ it follows that

$$
\boldsymbol{\Psi}_{i, j}^{(1)}+\boldsymbol{\Psi}_{i, j}^{(2)}+\boldsymbol{\Psi}_{i, j}^{(3)}+\cdots=\sum_{k=0}^{49} \boldsymbol{u}_{i, k}\left[\delta_{k, j}+\theta_{k, j}^{(1)}+\theta_{k, j}^{(2)}+\cdots\right] \boldsymbol{p},
$$

where we define $\delta_{k, j}=1$ for $j=k$ and $\delta_{k, j}=0$ for $j \neq k$. We may interpret (7.1) as follows. Recall that $\boldsymbol{I}+\boldsymbol{\Omega}=(\boldsymbol{I}-\boldsymbol{\Theta})^{-1}$ and $\boldsymbol{I}+\boldsymbol{B}=\left(\boldsymbol{I}-\boldsymbol{T}_{1}\right)^{-1}$. If we write $\boldsymbol{B}=\left[\boldsymbol{B}_{i, j}\right]$, where $\boldsymbol{B}_{i, j} \in \mathbb{R}^{51 \times 51}$ for each $i=0, \ldots, 49$ and $j=0, \ldots, 49$, and note that $\boldsymbol{B}=\boldsymbol{T}_{1}+\boldsymbol{T}_{1}^{2}+\boldsymbol{T}_{1}^{3}+\cdots$, then (7.1) can be interpreted as

$$
\boldsymbol{B}_{i, j}=\boldsymbol{U}_{i}(\boldsymbol{I}+\boldsymbol{\Omega})_{j} \boldsymbol{p},
$$

where $\boldsymbol{U}_{i}=\left[\boldsymbol{u}_{i, 0}, \cdots, \boldsymbol{u}_{i, 49}\right] \in \mathbb{R}^{51 \times 50},(\boldsymbol{I}+\boldsymbol{\Omega})_{j}=\left[\delta_{1, j}+\omega_{1, j}, \cdots, \delta_{m, j}+\omega_{m, j}\right]^{\top} \in \mathbb{R}^{50 \times 1}$, and $\boldsymbol{p} \in \mathbb{R}^{1 \times 51}$. Thus, the required matrix inversion is reduced to a collection of feasible matrix multiplications.

To find the invariant vector for $\boldsymbol{S}$ we note first that $\boldsymbol{S}$ is a stochastic matrix. That is, $\boldsymbol{S} \boldsymbol{f}=\boldsymbol{f}$ where $\boldsymbol{f}=[1, \ldots, 1]^{\top} \in \mathbb{R}^{50 \times 1}$. Thus we can use the very stable iteration $\boldsymbol{\rho}^{(r+1)}=\boldsymbol{\rho}^{(r)} \boldsymbol{S}$ for each $r \in \mathbb{N}$ with $\boldsymbol{\rho}^{(1)}=[1, \ldots, 1] / 50 \in \mathbb{R}$ to calculate the invariant vector $\rho$.

\subsection{Checking the new calculations}

For constant demand with $d=2$ we checked our answers using the Gaussian elimination described in [7]. We outline the crucial details below. Let $\psi_{r}=\pi_{m-r}$ for each $r=1,2, \ldots, m$. Define $W_{0}=(\boldsymbol{I}-\boldsymbol{A})^{-1}$ and use the recursive definitions

$$
\boldsymbol{W}_{s}=\left[\boldsymbol{I}-\boldsymbol{A} \boldsymbol{W}_{0} \Sigma \boldsymbol{W}_{1} \Sigma \cdots \boldsymbol{W}_{s-1} \Sigma\right]^{-1},
$$

for each $s=1, \ldots, d$, and

$$
\boldsymbol{W}_{s}=\left[\boldsymbol{I}-\boldsymbol{A} \boldsymbol{W}_{s-d} \Sigma \boldsymbol{W}_{s-d+1} \Sigma \cdots \boldsymbol{W}_{s-1} \Sigma\right]^{-1},
$$

for each $s=d+1, \ldots, m-1$. Let $\boldsymbol{X}_{m}=\boldsymbol{W}_{m-d} \Sigma \boldsymbol{W}_{m-d+1} \Sigma \cdots \boldsymbol{W}_{m-1}$ and define

$$
\boldsymbol{\varphi}_{r}=\boldsymbol{\pi}_{m} \boldsymbol{X}_{m} \Sigma^{-r+1} \boldsymbol{A},
$$

for $r=1,2, \ldots, d$, and

$$
\boldsymbol{\varphi}_{r}=\boldsymbol{\pi}_{m}\left\{\boldsymbol{X}_{m} \Sigma^{-r+1} \boldsymbol{A}-\Sigma^{-r+d} \boldsymbol{A}\right\},
$$

for $r=d+1, d+2, \ldots, m$. In [7] it has been shown using Gaussian elimination that the steady state equation (4.1) can be reduced to an equation of the form

$$
\psi(I+U)=\varphi
$$

where $\boldsymbol{U} \in \mathbb{R}^{m(n+1) \times m(n+1)}$ is a block upper triangular matrix with blocks $\boldsymbol{U}_{i j} \in \mathbb{R}^{(n+1) \times(n+1)}$ for each $i=0,1, \ldots, m-1$ and $j=0,1, \ldots, m-1$, such that $\boldsymbol{U}_{i j}=0$ for $i \leq j+d+1$ and $\boldsymbol{U}_{i j}=\Sigma^{-i+j+d+1} \boldsymbol{A}$ when $i \geq j+d+2$. Since $\boldsymbol{U}^{r}=0$ when $r(d+1)>m$ this equation is easily solved. Thus, we obtain an expression of the form

$$
\boldsymbol{\psi}=\boldsymbol{\varphi}(\boldsymbol{I}+\boldsymbol{U})^{-1} \quad \Longleftrightarrow \quad \pi_{s}=\pi_{m} \boldsymbol{R}_{s},
$$


for each $s=1, \ldots, m-1$. The final equation for the invariant level probabilities is

$$
\left[\sum_{s=0}^{d} \boldsymbol{\pi}_{s}\right] \boldsymbol{A}_{m}+\sum_{s=d+1}^{m} \boldsymbol{\pi}_{s} \boldsymbol{A}_{m+d-s}=\boldsymbol{\pi}_{m} .
$$

If we use the formula $\boldsymbol{\pi}_{s}=\boldsymbol{\pi}_{m} \boldsymbol{R}_{S}$ then the final equation becomes an eigenvector equation, i.e.

$$
\boldsymbol{\pi}_{m} \boldsymbol{R}=\boldsymbol{\pi}_{m}
$$

where $\boldsymbol{R} \in \mathbb{R}^{(n+1) \times(n+1)}$ is given by

$$
\boldsymbol{R}=\left[\sum_{s=0}^{d} \boldsymbol{R}_{s}\right] \boldsymbol{A}_{m}+\sum_{s=d+1}^{m-1} \boldsymbol{R}_{s} \boldsymbol{A}_{m+d-s}+\boldsymbol{A}_{d}
$$

We used MATLAB to find $\boldsymbol{\pi}_{m}$ from (7.2) up to an unknown positive multiple and hence determined the invariant level probabilities by back substitution.

\section{Extension to a series of three dams}

We outline briefly the extension of this method to a series of three dams-a capture dam where stormwater is captured from an urban watercourse, a holding dam where water is held while impurities are removed from suspension, and a distribution dam where water is collected for distribution. The state of the system is denoted by $(i, j, k) \in\{0,1, \ldots, \ell\} \times\{0,1, \ldots, m\} \times$ $\{0,1, \ldots, n\}$, where $i, j$, and $k$ are the current contents of the distribution, holding, and capture dams, respectively, and $\ell, m$, and $n$ are the capacities of the distribution, holding, and capture dams, respectively. The content of the distribution dam is the level, the content of the holding dam is the primary phase, and the content of the capture dam is the secondary phase. The states are ordered by the following rules:

(1) $(i, \cdot, \cdot) \prec(i+1, \cdot, \cdot)$,

(2) $(i, j, \cdot) \prec(i, j+1, \cdot)$,

(3) $(i, j, k) \prec(i, j, k+1)$.

Where necessary, we assume that $\ell \leq m \leq n$.

By considering the set of all elementary transitions it can be shown that the transition matrix for the fully stochastic system can be written in the form

$$
\boldsymbol{T}=\left[\begin{array}{ccccc}
\Phi_{0,0} \boldsymbol{A} & \Phi_{0,1} \boldsymbol{A} & \ldots & \Phi_{0, \ell-1} \boldsymbol{A} & \Phi_{0, \ell} \\
\Phi_{1,0} \boldsymbol{A} & \Phi_{1,1} \boldsymbol{A} & \ldots & \Phi_{1, \ell-1} \boldsymbol{A} & \Phi_{1, \ell} \\
\vdots & \vdots & \ddots & \vdots & \vdots \\
\Phi_{\ell-1,0} \boldsymbol{A} & \Phi_{\ell-1,1} \boldsymbol{A} & \ldots & \Phi_{\ell-1, \ell-1} \boldsymbol{A} & \Phi_{\ell-1, \ell} \\
\Phi_{\ell, 0} \boldsymbol{A} & \Phi_{\ell, 1} \boldsymbol{A} & \ldots & \Phi_{\ell, \ell-1} \boldsymbol{A} & \Phi_{\ell, \ell}
\end{array}\right]=\left[\begin{array}{cc}
\boldsymbol{T}_{1} & \boldsymbol{T}_{2} \\
\boldsymbol{T}_{3} & \Phi_{\ell, \ell}
\end{array}\right],
$$

where the matrices $\Phi_{i, j} \in \mathbb{R}^{(m+1)(n+1) \times(m+1)(n+1)}$ are defined by the formulae

$$
\Phi_{i, j}= \begin{cases}q_{i}^{+} \Sigma^{j}+\sum_{r=1}^{\min (i, j)} q_{i-r} \Sigma^{j-r} & \text { for } 0 \leq j<\ell, \\ q_{i}{ }^{+} \boldsymbol{A}_{\ell}+\sum_{r=1}^{i} q_{i-r} \boldsymbol{A}_{\ell-r} & \text { for } j=\ell,\end{cases}
$$


The matrices $\boldsymbol{A}, \boldsymbol{\Sigma} \in \mathbb{R}^{(m+1)(n+1) \times(m+1)(n+1)}$ are given by

$$
\boldsymbol{A}=\left[\begin{array}{ccccc}
\boldsymbol{P} & \boldsymbol{\Upsilon} \boldsymbol{P} & \ldots & \boldsymbol{\Upsilon}^{m-1} \boldsymbol{P} & \boldsymbol{P}_{m} \\
0 & 0 & \ldots & 0 & 0 \\
\vdots & \vdots & \ddots & \vdots & \vdots \\
0 & 0 & \ldots & 0 & 0
\end{array}\right], \quad \boldsymbol{\Sigma}=\left[\begin{array}{ccccc}
0 & 0 & \ldots & 0 & \boldsymbol{I} \\
\boldsymbol{I} & 0 & \ldots & 0 & 0 \\
\vdots & \vdots & \ddots & \vdots & \vdots \\
0 & 0 & \cdots & 0 & \boldsymbol{I}
\end{array}\right]
$$

and $\boldsymbol{A}_{i} \in \mathbb{R}^{(m+1)(n+1) \times(m+1)(n+1)}$ is given by

$$
\boldsymbol{A}_{i}=\left[\begin{array}{ccccccc}
0 & 0 & \ldots & 0 & \ldots & 0 & 0 \\
\vdots & \vdots & & \vdots & & \vdots & \vdots \\
0 & 0 & \ldots & 0 & \ldots & 0 & 0 \\
\boldsymbol{P} & \boldsymbol{\Upsilon} \boldsymbol{P} & \ldots & \boldsymbol{\Upsilon}^{m-i} \boldsymbol{P} & \ldots & \boldsymbol{\Upsilon}^{m-1} \boldsymbol{P} & \boldsymbol{P}_{m} \\
0 & \boldsymbol{P} & \ldots & \boldsymbol{\Upsilon}^{m-i-1} \boldsymbol{P} & \ldots & \boldsymbol{\Upsilon}^{m-2} \boldsymbol{P} & \boldsymbol{P}_{m-1} \\
\vdots & \vdots & \ddots & \vdots & & \vdots & \vdots \\
0 & 0 & \ldots & \boldsymbol{P} & \ldots & \boldsymbol{\Upsilon}^{i-1} \boldsymbol{P} & \boldsymbol{P}_{i}
\end{array}\right]=\left[\begin{array}{c}
0 \\
\vdots \\
0 \\
\boldsymbol{G} \\
\delta \boldsymbol{G} \\
\vdots \\
\delta^{m-i} \boldsymbol{G}
\end{array}\right]
$$

for each $i=0,1, \ldots, \ell$. The matrices $\boldsymbol{P}, \Upsilon \in \mathbb{R}^{(n+1) \times(n+1)}$ are defined by

$$
\boldsymbol{P}=\left[\begin{array}{ccccc}
p_{0} & p_{1} & \cdots & p_{n-1} & p_{n}{ }^{+} \\
0 & 0 & \cdots & 0 & 0 \\
\vdots & \vdots & \ddots & \vdots & \vdots \\
0 & 0 & \cdots & 0 & 0
\end{array}\right], \quad \Upsilon=\left[\begin{array}{ccccc}
0 & 0 & \cdots & 0 & 1 \\
1 & 0 & \cdots & 0 & 0 \\
\vdots & \vdots & \ddots & \vdots & \vdots \\
0 & 0 & \cdots & 0 & 1
\end{array}\right]
$$

and $\boldsymbol{P}_{j} \in \mathbb{R}^{(n+1) \times(n+1)}$ is given by

$$
\boldsymbol{P}_{j}=\left[\begin{array}{ccccccc}
0 & 0 & \cdots & 0 & \cdots & 0 & 0 \\
\vdots & \vdots & & \vdots & & \vdots & \vdots \\
0 & 0 & \cdots & 0 & \cdots & 0 & 0 \\
p_{0} & p_{1} & \cdots & p_{n-j} & \cdots & p_{n-1} & p_{n}{ }^{+} \\
0 & p_{0} & \cdots & p_{n-j-1} & \cdots & p_{m-2} & p_{n-1} \\
\vdots & \vdots & \ddots & \vdots & & \vdots & \vdots \\
0 & 0 & \cdots & p_{0} & \cdots & p_{j-1} & p_{j}^{+}
\end{array}\right]=\left[\begin{array}{c}
\mathbf{0} \\
\vdots \\
\mathbf{0} \\
\boldsymbol{p} \\
\delta \boldsymbol{p} \\
\vdots \\
\delta^{n-j} \boldsymbol{p}
\end{array}\right]
$$

We consider a censored process with transition matrix $S=\Phi_{\ell, \ell}+\boldsymbol{T}_{3}\left(\boldsymbol{I}-\boldsymbol{T}_{1}\right)^{-1} \boldsymbol{T}_{2}$ by observing only at level $\ell$ to find an invariant probability $\boldsymbol{\rho}=\left(\boldsymbol{\rho}_{0}, \boldsymbol{\rho}_{1}, \ldots, \boldsymbol{\rho}_{m}\right) \in \mathbb{R}^{1 \times(m+1)(n+1)}$. Since $\rho_{r} \in \mathbb{R}^{1 \times(n+1)}$ is the relative invariant probability of the secondary phases given that the level is $\ell$ and the primary phase is $r$, it follows that $\pi_{\ell, r}=\alpha_{\ell, r} \rho_{r}$ where the constant $\alpha_{\ell, r} \in[0,1]$ is the total invariant probability for level $\ell$ and primary phase $r$.

For $i=0,1, \ldots, \ell$ let $\boldsymbol{E}_{i} \in \mathbb{R}^{(m+1)(n+1) \times(n+1)}$ denote the unit block column vector with $\boldsymbol{I} \in \mathbb{R}^{(n+1) \times(n+1)}$ in the $i$ th position and $0 \in \mathbb{R}^{(n+1) \times(n+1)}$ elsewhere. Now, $\Sigma^{i} A=\boldsymbol{E}_{i} \boldsymbol{G}$, where $\boldsymbol{G}=\left[\boldsymbol{P}, \Upsilon \boldsymbol{P}, \ldots, \Upsilon^{m-1} \boldsymbol{P}, \boldsymbol{P}_{m}\right]$; hence, $\Phi_{i, j} A=\boldsymbol{U}_{i, j} \boldsymbol{G}$, where $\boldsymbol{U}_{i, j} \geq 0$. Thus, we can simplify the expressions for $\boldsymbol{T}_{1}$ and $\boldsymbol{T}_{3}$ by writing

$$
\boldsymbol{T}_{1}=\left[\boldsymbol{U}_{i, j} \boldsymbol{G}\right], \quad \boldsymbol{T}_{3}=\left[\boldsymbol{U}_{\ell, j} \boldsymbol{G}\right] .
$$

Hence, $\boldsymbol{T}_{1}^{s}=\left[\boldsymbol{U}_{i, j}^{(s)} \boldsymbol{G}\right]$ for each $s \in \mathbb{N}$, where $\boldsymbol{U}_{i, j}^{(s)} \geq 0$, and $\boldsymbol{R}=\sum_{s=1}^{\infty} \boldsymbol{T}_{1}^{s}$ can be written in the form $\boldsymbol{R}=\left[\boldsymbol{V}_{i, j} \boldsymbol{G}\right]$ where $\boldsymbol{V}_{i, j} \geq 0$. Once again we rearrange the required matrix 
multiplications by defining nonnegative block elements $\boldsymbol{\Theta}_{i, j}=\boldsymbol{G} \boldsymbol{U}_{i, j}$ and $\boldsymbol{\Omega}_{i, j}=\boldsymbol{G} \boldsymbol{V}_{i, j}$ for each $i, j=0,1, \ldots, \ell-1$ and corresponding new matrices $\boldsymbol{\Theta}=\left[\boldsymbol{\Theta}_{i, j}\right]$ and $\boldsymbol{\Omega}=\left[\boldsymbol{\Omega}_{i, j}\right]$. These matrices satisfy similar key relationships to the analogous matrices in the smaller system. We have

$$
\boldsymbol{U}_{i, j} \boldsymbol{G}=\left[\begin{array}{c}
\boldsymbol{U}_{i, j, 0} \\
\boldsymbol{U}_{i, j, 1} \\
\vdots \\
\boldsymbol{U}_{i, j, m}
\end{array}\right]\left[\begin{array}{lllll}
\boldsymbol{e}_{0} \boldsymbol{p} & \boldsymbol{e}_{1} \boldsymbol{p} & \cdots & \boldsymbol{e}_{m-1} \boldsymbol{p} & \sum_{s=m}^{n} \boldsymbol{e}_{s} \delta^{s-m} \boldsymbol{p}
\end{array}\right],
$$

where $\boldsymbol{U}_{i, j, r}=\left[\boldsymbol{u}_{i, j, r, 0}, \boldsymbol{u}_{i, j, r, 1}, \ldots, \boldsymbol{u}_{i, j, r, n}\right] \in \mathbb{R}^{(n+1) \times(n+1)}$ and $\boldsymbol{u}_{i, j, r, s} \in \mathbb{R}^{(n+1) \times 1}$. The steady-state equation $\boldsymbol{\pi} \boldsymbol{T}=\boldsymbol{\pi}$ now gives

$$
\sum_{i=0}^{\ell} \sum_{r=0}^{m} \boldsymbol{\pi}_{i, r} \boldsymbol{u}_{i, j, r, s} \boldsymbol{p}=\boldsymbol{\pi}_{j, s} \quad \Longleftrightarrow \quad \alpha_{j, s} \boldsymbol{p}=\boldsymbol{\pi}_{j, s},
$$

where $\alpha_{j, s} \geq 0$, for each $j=0,1, \ldots, \ell-1$ and $s=0,1, \ldots, m-1$, and

$$
\sum_{i=0}^{\ell} \sum_{r=0}^{m} \boldsymbol{\pi}_{i, r} \sum_{s=m}^{n} \boldsymbol{u}_{i, j, r, s} \delta^{s-m} \boldsymbol{p}=\boldsymbol{\pi}_{j, m} \quad \Longleftrightarrow \quad \sum_{s=m}^{n} \beta_{j, s} \delta^{s-m} \boldsymbol{p}=\boldsymbol{\pi}_{j, m},
$$

where $\beta_{j, s} \geq 0$, for each $j=0,1, \ldots, \ell-1$ and $s=m, m+1, \ldots, n$. Thus, we observe that the steady state probability $\boldsymbol{\pi}_{j, s}$ is simply a multiple of the supply probability $\boldsymbol{p}$ for each $j=0,1, \ldots, \ell-1$ and $s=0,1, \ldots, m-1$. This is a key observation. Note too that $\alpha_{j, m}=\sum_{s=m}^{n} \beta_{j, s}$. Define $x_{j, r, s}=\rho_{r} \boldsymbol{u}_{\ell, j, r, s}$ for each $r=0,1, \ldots, m$ and $s=0,1, \ldots, n$. If we substitute for $\boldsymbol{\pi}_{i, r}$ and $\boldsymbol{\pi}_{i, m}$ into the left-hand sides of the long form of (8.1) and (8.2), respectively, and define $\boldsymbol{\alpha}=\left(\boldsymbol{\alpha}_{0}, \ldots, \boldsymbol{\alpha}_{\ell-1}\right)$, where $\boldsymbol{\alpha}_{j}=\left(\alpha_{j, 0}, \ldots, \alpha_{j, m-1}, \beta_{j, m}, \ldots, \beta_{j, n}\right)$, and use $\pi_{\ell, r}=\alpha_{\ell, r} \rho_{r}$, then we can combine (8.1) and (8.2) into a single system of equations, i.e.

$\boldsymbol{\alpha}_{j}=\sum_{i=0}^{\ell-1} \boldsymbol{\alpha}_{i} \boldsymbol{\Theta}_{i, j}+\boldsymbol{\alpha}_{\ell} \boldsymbol{X}_{j} \quad \Longleftrightarrow \quad \boldsymbol{\alpha}=\boldsymbol{\alpha} \boldsymbol{\Theta}+\boldsymbol{\alpha}_{\ell} \boldsymbol{X} \quad \Longleftrightarrow \quad \boldsymbol{\alpha}=\boldsymbol{\alpha}_{\ell} X(I-\boldsymbol{\Theta})^{-1}$,

where we have defined $\boldsymbol{X}_{j}=\left[x_{j, r, s}\right] \in \mathbb{R}^{(m+1) \times(n+1)}$, for each $j=0,1, \ldots, \ell-1$, and $\boldsymbol{X}=\left[\boldsymbol{X}_{1}, \ldots, \boldsymbol{X}_{\ell-1}\right]$. The algebra required to derive (8.3) is complicated but essentially routine and uses the relationships $\boldsymbol{\theta}_{i, j, r}=\boldsymbol{p} \boldsymbol{U}_{i, j, r}$, for $r=0,1, \ldots, m-1$, and $\boldsymbol{\theta}_{i, j, r}=\delta_{r-m} \boldsymbol{p} \boldsymbol{U}_{i, j, m}$, for $r=m, m+1, \ldots, n$. We also use the independence of $\boldsymbol{p}, \delta \boldsymbol{p}, \ldots, \delta^{n} \boldsymbol{p}$. It is relatively straightforward to extract expressions for the invariant probabilities from (8.3). We have not yet looked for the most efficient numerical calculation schemes nor considered any specific examples.

\section{Conclusions}

We have established analytic expressions for the steady state level and phase probabilities in a system of two dams with stochastic daily supply and independent stochastic daily demand. Our analysis extends previous work on a similar system in which demand is constant. In each case the key solution idea is to reduce the size of the matrix computations by one order of magnitude - that is, to the size required for consideration of a single dam. In this paper we go 
further and establish important characteristics of the invariant vectors for the various different levels. In particular, the invariant vector for the top level is cast as the invariant vector of a censored process involving only phase transitions at the top level. The invariant vectors for phase transitions at all lower levels are shown to be scalar multiples of the supply probability vector. The advantage in this characterisation of the process is that it apparently paves the way for an extension of the analysis to systems of more than two dams although details of the general analysis have not yet been considered.

\section{Dedication}

This paper is dedicated to our co-author, friend, and colleague, Charles E. M. Pearce, who died on 8th of June 2012, as a result of a tragic car accident at the Manakaiua River on the West Coast of the South Island of New Zealand. Charles was a most generous and agreeable person and an outstanding applied mathematician. He won the ANZIAM medal in 2001 and the ASOR medal in 2007. Charles was the Sir Thomas Elder Professor of Mathematics at The University of Adelaide. He is sadly missed by his family, friends, and colleagues.

\section{References}

[1] Archibald, T. W., McKinnon, K. I. M. and Thomas, L. C. (1997). An aggregate stochastic dynamic programming model of multireservoir systems. Water Resources Res. 33, 333-340.

[2] Archibald, T. W., McKinnon, K. I. M. and Thomas, L. C. (2006). Modeling the operation of multireservoir systems using decomposition and stochastic dynamic programming. Naval Res. Logistics 53, 217-225.

[3] Archibald, T. W., Buchanan, C. S., Thomas, L. C. and McKinnon, K. I. M. (2001). Controlling multireservoir systems. Europ. J. Operat. Res. 129, 619-626.

[4] Cohen, M. J. And Brown, M. T. (2007). A model examining hierarchical wetland networks for watershed stormwater management. Ecological Modelling 201, 179-193.

[5] Gani, J. (1969). Recent advances in storage and flooding theory. Adv. Appl. Prob. 1, 90-110.

[6] Gaver, D. P., Jacobs, P. A. And Latouche, G. (1984). Finite birth-and-death models in randomly changing environments. Adv. Appl. Prob. 16, 715-731.

[7] Howlett, P. and Piantadosi, J. (2009). An application of the pump-to-fill policy for management of urban stormwater. Environ. Modeling Assessment 14, 195-207.

[8] Latouche, G. and Ramaswami, V. (1999). Introduction to Matrix Analytic Methods in Stochastic Modeling. Society for Industrial and Applied Mathematics, Philadelphia, PA.

[9] Latouche, G., Jacobs, P. A. And Gaver, D. P. (1984). Finite Markov chain models skip-free in one direction. Naval Res. Logistics Quart. 31, 571-588.

[10] Moran, P. A. P. (1954). A probability theory of dams and storage systems. Austral. J. Appl. Sci. 5, $116-124$.

[11] Moran, P. A. P. (1959). The Theory of Storage. Methuen, London.

[12] Neuts, M. F. (1981). Matrix-Geometric Solutions in Stochastic Models. An Algorithmic Approach. Johns Hopkins University Press, Baltimore, MD.

[13] Neuts, M. F. (1989). Structured Stochastic Matrices of $M / G / 1$ Type and Their Applications. Marcel Dekker, New York.

[14] Pearce, C. E. (2009). Optimal stormwater management with two dams: the dynamics of the pump-to-fill policy. Optimization 58, 181-192.

[15] Pearce, C. E. M., Piantadosi, J. and Howlett, P. G. (2007). On an optimal control policy for stormwater management in two connected dams. J. Industrial Manag. Optimization 3, 313-320.

[16] Piantadosi, J. (2004). Optimal policies for storage of urban stormwater. Doctoral Thesis, University of South Australia. Available at http://arrow.unisa.edu.au:8081/1959.8/82105.

[17] Piantadosi, J., Howlett, P. G., Bean, N. G. and Beecham, S. (2010). Modelling systems of reservoirs using structured Markov chains. Proc. Inst. Civ. Eng. Water Manag. 163, 407-416.

[18] Rosenberg, K., Boland, J. And Howlett, P. G. (2004). Simulation of monthly rainfall totals. ANZIAM J. 46, E85-E104.

[19] Thomas, P., Howlett, P. G. and Piantadosi, J. (2010). Investigating the optimal management of the Helps Road drain urban stormwater harvesting system within the city of Salisbury. Europ. Water 29, 11-19.

[20] Yeo, G. F. (1974). A finite dam with exponential release. J. Appl. Prob. 11, 122-133.

[21] Yeo, G. F. (1975). A finite dam with variable release rate. J. Appl. Prob. 12, 205-211. 